\title{
Research on Text Emotion Analysis Method Based on Attention Mechanism and BGRU Network
}

\author{
Guangjun Liu*, Weiqing Yang, Lining Zhan, Xiangjun Li \\ School of Information Engineering, Xi'an University, Shaanxi Xi’an, 710065, China
}

Keywords: Attention Mechanism, BGRU, Text Emotion.

\begin{abstract}
The rapid development of the Internet makes social software such as e-commerce, major portals and other social software and new media emerge in endlessly, which has gradually become an important platform for people to exchange information and obtain information. Web text generally has the user's rich emotion, needs to carry on the data mining to these rich emotion, therefore this proposed to carry on the text emotion analysis research based on the attention mechanism and the double-door circular neural network model, is based on the two-way GRU emotion analysis algorithm model, unifies the emotion analysis character to carry on the algorithm optimization improvement, the improved algorithm model can enhance the Web text emotion analysis effect significantly.
\end{abstract}

\section{Introduction}

With the continuous development of Internet technology and artificial intelligence technology, the emergence and wide application of a large number of social media tools have greatly changed people's traditional communication methods, people can use these communication platforms to achieve emotional communication and web text information cycle, the more typical of which include Facebook, Twitter and so on. Web emotional analysis can generally be divided into two types: text level and short text emotional analysis, from the upper limit of affective polarity can be divided into positive and negative. The Web text emotion analysis method based on machine learning can carry out the feature extraction of test expectation and training times, and realize the analysis and research of emotion data through machine learning algorithm. This research, can carry on the emotion data analysis, has the certain practical value, for example according to the user's consumption habit, the enterprise may according to the user feedback and the product performance improvement, can guide the social production.

\section{Research Status}

The development and promotion of social media allow users to establish new network relationships through the Internet, some domestic research through the detection of user friend relationships to understand and study the structured information based on social media, in the introduction of attention mechanism can better carry out the analysis of internal attributes.[1]The attention mechanism is introduced into the sentence sorting model, which can help to capture the semantic logical relation of sentences.[2]In the traditional text emotion classification, it is difficult to express the context semantic information effectively considering the emotion semantics, without paying attention to the importance of sentence meaning. Therefore, in this case, the two-way gate loop unit network emotion analysis method can replace the previous simple network and effectively combine the context semantics.[3]Text emotion analysis is called opinion mining, which is to use text mining technology, natural language processing technology to extract the information of the supervisor in the original material.[4]there are significant differences between general affective analysis and web text affective analysis, which are generated by internet carriers. in addition to information independence, web text affective analysis has very strong arbitrariness, immediacy, simplicity and arbitrariness. 


\section{Self-Attention Mechanism}

\subsection{Self-Attention Mechanism Basis}

Self-attention is also called internal attention, and the self-attention mechanism represents a kind of attention mechanism with direct correlation with different positions in the sequence. In recent years, internet technology and computer technology have promoted the development of deep learning technology, and the self-attention mechanism has also been widely used in this context. In google's machine translation model, the self-attention model has been widely used, and it has achieved very good results. In addition, many people apply the self-attention mechanism to the statement direction quantization, which can achieve better application effect on multiple common data sets with higher accuracy. In general, the input content of the source data is different from the output content of the target data in the coding-decoding framework of the regular task. In machine translation, if the input source data is a Chinese sentence, then the output target data may be based on the needs of the customer in English or other languages. The self-attention mechanism can be regarded as a special attention mechanism in which the source data and the target data are merged into one.

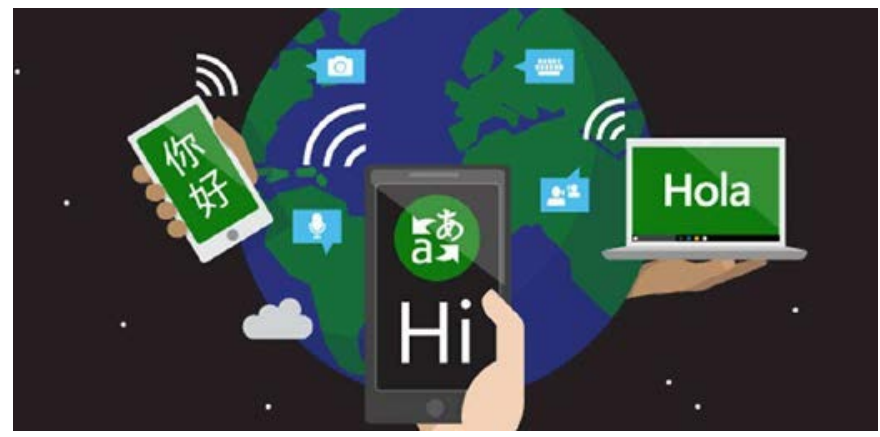

Figure 1 Machine translation

\subsection{Self-Attention Mechanism Calculation Steps}

The calculation step of the self-attention mechanism can be divided into three steps, the first step is to analyze and calculate the similarity between an element of the target data or object and the key word, followed by the normalization of all the original scores, and finally the weight coefficient can be summed up to calculate the self-attention value of the element. In the first step, the corresponding function and calculation mechanism will be introduced according to the query needs and the set keywords. the second step is to convert the first step to calculate the obtained fractional values and obtain the corresponding weight coefficients to achieve the normalization of the values. The third step is to obtain the self-attention value by using the weighted numerical summation. When the self-attention mechanism is introduced, it is more convenient to acquire the long-range interdependent features to capture sentences, because the self-attention mechanism can directly connect any two words in a sentence using one step. This method can greatly shorten the distancedependent feature distance, which cannot only help the computation parallelism, but also make it more convenient. Because of these advantages of the self-attention mechanism, it is more widely used in the field of natural language processing and artificial intelligence.

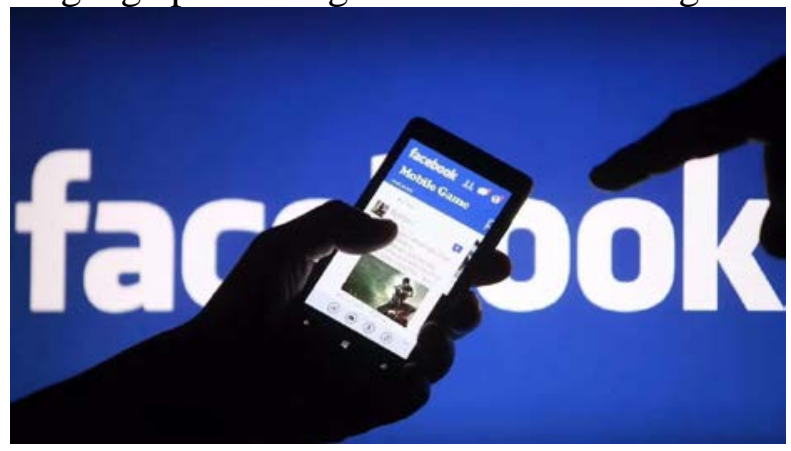

Figure 2 Facebook 


\section{Text Affective Analysis of Bgru Networks}

\subsection{Two-Way Door Cycle Unit}

In the traditional cyclic neural network model, the data processing of any length sequence can be carried out, and the input information of any time mentioned above can be preserved in the network, which can be very superior to the combination of artificial neural networks in natural language processing. Because there are many problems in the traditional RNN model design, there is the phenomenon of gradient dispersion in the training process, so it needs to take more time, and the effect of training is difficult to meet what people want. in order to solve the defects in the existing patterns, the short-and-short-term memory network and the threshold cycle unit are proposed. the gru can save more time because of its simple, efficient and flexible structure design, and it is more widely used in deep learning. In general, the one-way GRU model in timing processing can only memorize the data content of the current moment, without paying attention to the future text information, which will make the performance of the whole model decrease and have a negative effect on the final effect. In this case, it is necessary to propose a two-way GRU model structure to realize the algorithm optimization design and improve the model performance and classification effect in an all-round way.

\subsection{Optimal Design of Algorithm}

introducing a self-attention mechanism to optimize and improve the RNN model can improve the performance of the model. In the depth learning environment, the model weight matrix and bias parameters are initialized, which will have a direct effect on the classification effect. The Web uses an English text data set, so we need to use the clean_str tool and the Beautiful Soup tool to preprocess the data, and then use word2vec to construct the Web text word vector. Word2vec belongs to the statistical method of effectively learning the text corpus embedding independent words, the key idea of which is to use vectors to represent each word on the basis of context and then to learn vector parameters by predicting the objective function. One of the core technologies in web text emotion analysis is feature fusion, which will have a direct effect on the final result, and can be divided into two types: direct and indirect feature fusion. The former refers to the integration of data based on the combination of pre-training sub-vector machine pre-processing text, and finally transforms into a word vector matrix to train the model. indirect feature fusion can use machine learning and deep learning models to achieve text data training and extract feature data.

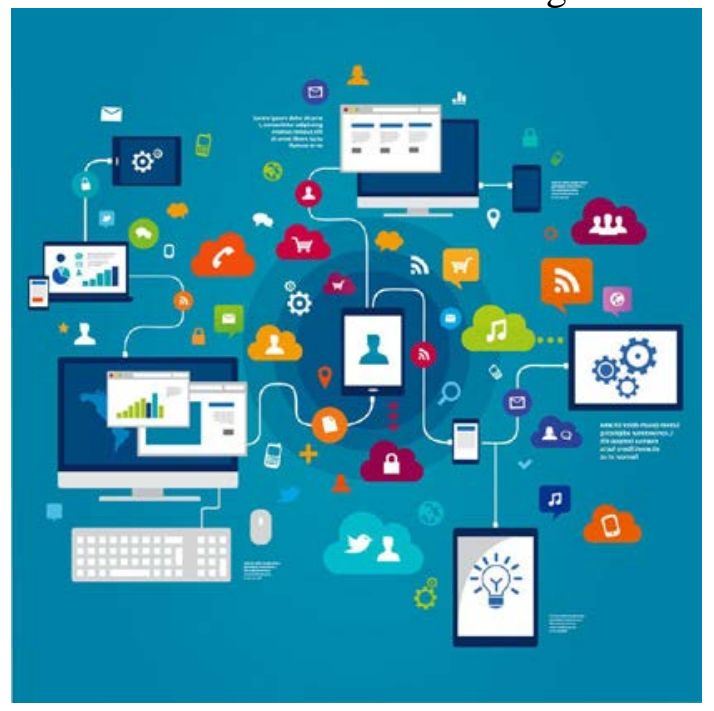

Figure 3 All kinds of social media

The improved model is divided into two layers, the first layer and the second layer are the data input layer and the sneak layer respectively. In the training of neural network model, we need to pay attention to the selection and setting of parameters, and there will be significant differences in the selection of different parameters training results. In deep learning, the training goal of neural 
network model is to realize the minimum model of choosing loss function. In the training optimization of objective function, the error measure should be minimized. the error of 0 indicates that the model is perfect, if the error deviates from 0 , then the model is not perfect, the closer the loss function value is to the performance of the 0 model, and the greater the deviation from 0 , the worse the model performance. In order to realize the optimization improvement of the algorithm, the model adopts the small batch gradient descent method, divides the training data into batches, and then arranges the update of the parameters, which can improve the training effect while reducing the randomness of the model and greatly reduce the calculation amount of the model. the better performance rmsprop optimizerizer is used in the circulating neural network, and it is also especially suitable for the task of natural meter processing of emotion classification. when the specific training is carried out, the parameters of the optimizer will keep the default value unchanged.

\section{Conclusion}

In the field of natural language processing, web text emotion analysis has gradually become a research hotspot, coupled with the continuous development of artificial intelligence technology, deep learning model is more widely used in image recognition, speech recognition and text processing, and has achieved very good results. Using attention mechanism learning and deep learning algorithm to analyze the text emotion problem, we can get a good result of emotion classification, and can get a very good performance on the common emotion data set.

\section{Acknowledgements}

This work is supported by the Science and Technology Plan Project of Xi'an (No. 2017CGWL35, No. 2016CXWL22, No. 2016CXWL21).

\section{References}

[1] Zhao, Yun., Wu, Fan., Wang, Zhongqing., etal. User relationship extraction based on attention mechanism and text information. Chinese Journal of Information, vol. 33, no. 3, pp. 1-7, 2018.

[2] Luan, Kexin., Du, Xinkai., Sun, Chengjie., et al. Method of sentence sorting based on attention mechanism. Chinese Journal of Information, vol. 32, no. 1, 2018.

[3] Yin, Liangliang., Sun, Hongguang., Wang, Chao., et al. Research on text emotion analysis method based on attention mechanism and BGRU network. Wireless Interconnection Technology, no. 9, pp. 27-29, 2019.

[4] Sun, Yan., Zhou, Xueguang., Fu, Wei. Unsupervised text emotion analysis based on topic emotion mixing model. Journal of Peking University (Natural Science Edition),vol. 49, no. 1, pp. 102-108, 2013. 